\title{
Detecção precoce de neoplasias cutâneas em cuidados de saúde primários Relato de uma experiência
}

Ana Cristina Neves, ${ }^{*}$ Gustavo Melo Ferreira, ${ }^{*}$ Maria da Conceição Teixeira, ${ }^{* *}$ Eugénia Monteiro***

\section{RESUMO}

Introdução: As neoplasias cutâneas são frequentes em Portugal. Estima-se para o melanoma uma incidência de 8/100.000 hab/ano e para os epiteliomas uma incidência de 100/100.000 hab/ano. A incidência do melanoma tem aumentado em 6 a $7 \%$ ao ano. Vinte por cento dos doentes com melanoma morrerão da doença e metade destes com menos de 40 anos. Este aumento deve-se não só ao surgimento de novos casos, mas também à maior procura de serviços.

Metodologia: Este trabalho relata uma experiência ao longo de 22 meses (Julho/2008 a Maio/2010) de exérese de lesões cutâneas com recurso a procedimentos invasivos mínimos. Foram intervencionados 282 utentes, dos quais 164 (58\%) do sexo feminino, incluídos em todas as faixas etárias.

Resultados: Foram realizados 341 procedimentos, dos quais 170 (49,8\%) com recurso a exame histológico para confirmação diagnóstica. As lesões mais comuns foram: quisto epidérmico, nevo melanocítico dérmico e nevo melanocítico composto. Foram também identificadas as seguintes lesões pré-malignas: queratose solar actínica, corno cutâneo, lesão induzida por HPV, nevo melanocítico atípico e nevo melanocítico congénito. Além disso, encontraram-se as seguintes lesões malignas: carcinoma espino-celular in situ, carcinoma baso-celular e melanomas. Em todos os casos de lesões pré-malignas foi confirmada histologicamente a exérese com margens livres e todos os casos de lesões malignas foram encaminhados aos cuidados secundários.

Discussão: Esta experiência mostra que é possível estender a detecção precoce e o tratamento do cancro cutâneo aos cuidados de saúde primários. A relação de proximidade deste serviço foi muito bem aceite pelos utentes, pois encurtou distâncias e tempos de espera. Será de considerar no futuro o estudo do custo-eficácia e impacto na saúde da população.

Palavras-chave: Procedimentos Cirúrgicos Menores; Neoplasias Cutâneas.

\section{INTRODUÇÃO}

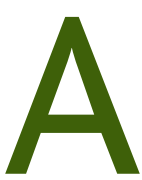

s neoplasias cutâneas constituem a forma mais frequente de neoplasia maligna na população de pele branca. Portugal, devido a sua grande exposição solar ao longo do ano, tem elevada prevalência, causando acentuada morbilidade e mortalidade. Apesar dos dados oficiais serem pouco rigorosos, os números disponibilizados no Instituto Português de Oncologia (IPO) permitem estimar a incidência de neoplasias cutâneas. De acordo com a Liga Portuguesa contra o Cancro, estima-se para o melanoma uma incidência de 8/100.000 hab/ano e para os

\footnotetext{
*Assistente de Medicina Geral e Familiar, USF S. Gonçalo, Amarante.

**Enfermeira Graduada, USF S. Gonçalo, Amarante.

***Interna de Medicina Geral e Familiar, USF S. Gonçalo, Amarante.
}

epiteliomas uma incidência de 100/100.000 hab/ano. A incidência do melanoma tem vindo a aumentar em cerca de 6 a $7 \%$ ao ano. Actualmente é o segundo mais frequente nas mulheres dos 20 aos 29 anos (depois do cancro da tiróide) e é o segundo mais frequente nas mulheres dos 30-39 anos (depois do cancro da mama). Um em cada cinco doentes com melanoma morrerá da doença e metade destes com menos de 40 anos. Este progressivo aumento deve-se não só a um aumento real de casos novos, mas também a uma maior procura de serviços e a um diagnóstico mais precoce. ${ }^{1,2}$

Ainda que os esforços educacionais para a prevenção primária de neoplasias cutâneas tenham contribuído para um maior conhecimento da população sobre o cancro de pele e a importância do uso protectores solares, 
houve alterações mínimas relativamente ao bronzeamento e exposição solar. Na verdade, aumentou a incidência de queimaduras solares e da prática de bronzeamento artificial. Estas conclusões resultaram do $59^{\circ} \mathrm{en}-$ contro da American Academy of Dermatology. Além disso, a eficácia dos protectores solares na prevenção dos diversos tipos de neoplasias cutâneas é ainda controversa; parece certo que previnem a queimadura solar e o carcinoma espino-celular, não sendo possível retirar conclusões da sua eficácia preventiva quanto ao carcinoma baso-celular e ao melanoma. Por outro lado, o seu uso pode transmitir uma falsa sensação de segurança, levando a uma exposição intencional prolongada. ${ }^{3}$

Embora o diagnóstico e tratamento precoces sejam fundamentais para melhorar o prognóstico e reduzir a mortalidade, ainda permanece controversa a utilidade dos programas de rastreio em massa. A U.S. Preventive Services Task Force (na sua revisão de Fevereiro de 2009) considera que falta evidência científica para recomendar quer o exame da pele de rotina por médico, quer o auto-exame da pele (recomendação de grau I). Esta opinião é partilhada por outras organizações, nomeadamente a Canadian Task Force on Preventive Health Care, a American Academy of Family Physician e a Physician Data Query. Entretanto, a American Cancer Society e a American College of Preventive Medicine recomendam o exame de rotina de toda a superfície corporal pelo médico e o auto-exame mensal. O maior problema dos testes de rastreio tem a ver com a acuidade do diagnóstico clínico. A U.S. Preventive Services Task Forcejustifica a sua recomendação com o facto de não haver estudos randomizados que avaliem a associação do rastreio clínico a um melhor prognóstico do cancro cutâneo. A possibilidade de que o tratamento precoce, como resultado do rastreio, melhore o prognóstico depende de uma evidência indirecta. ${ }^{4,5}$

A exérese de lesões cutâneas suspeitas para posterior exame histológico pode ser feita através do recurso a técnicas simples de pequena cirurgia (procedimentos cirúrgicos menores, cirurgia de ambulatório minor). Embora não exista um consenso internacional para a sua definição, a Associação Portuguesa de Cirurgia de Ambulatório (APCA) propõe no seu Enquadramento Nacional e Internacional a seguinte definição: «Toda a intervenção cirúrgica que, embora executada em condições de segurança e assepsia, possa dispensar a sua realização numa sala de bloco operatório (embora essa prática seja desejável), não exige mudança completa de roupa (cirurgião e doente), seja executada sem ajuda directa de um ajudante, sob anestesia local, não excedendo regra geral, 20 minutos, e sem necessidade de cuidados especiais de recobro». ${ }^{6}$ Os Cuidados de Saúde Primários constituem o nível assistencial mais próximo da população, onde devem ser orientados a maior parte dos problemas de saúde. A pequena cirurgia representa uma importante ferramenta de diagnóstico e tratamento que pode estar mais acessível e próxima da população. Os médicos de família, sob uma correcta capacitação e condições sanitárias adequadas, podem desempenhar um importante papel com elevado custo-eficácia. ${ }^{7,8}$

Este trabalho pretende relatar uma experiência ao longo de 22 meses (Julho 2008 a Maio 2010) de prática de exérese de lesões cutâneas e detecção de neoplasias cutâneas com recurso a procedimentos cirúrgicos menores (pequena cirurgia) no Centro de Saúde de Amarante (USF S. Gonçalo).

\section{METODOLOGIA}

\section{Organização do serviço}

Inicialmente os procedimentos foram realizados numa vulgar sala de tratamentos de enfermagem. Posteriormente, foi disponibilizada uma sala especificamente para o efeito com condições elementares (soalho e paredes laváveis, iluminação natural e artificial dirigível e condicionamento térmico do ambiente). A sala possui também equipamento essencial, nomeadamente, mesa operatória com acessibilidade em ambos lados, altura ajustável e superfície lavável, lavatório para as mãos, recipientes para lixo contaminado accionado por pedal, recipiente para objectos cortantes, carrinho de apoio e armário para consumíveis.

Os procedimentos foram agendados em horário específico. Os utentes sem médico ou de outras listas foram intervencionados em horário de consulta de reforço, também com agendamento prévio. Atendendo à disponibilidade de agendamento e de instrumental cirúrgico, foi estabelecido um limite de cinco intervenções por semana.

\section{Técnica utilizada}

A remoção de nevos e outros «sinais» foi feita por exérese elíptica com 1-2 $\mathrm{mm}$ de pele normal circundante. 
Não foi usado em nenhum caso a técnica de exérese cilíndrica (punch). O encerramento foi feito com sutura não absorvível e em alguns casos com sutura cutânea adesiva («steri-strip») para melhor efeito cosmético. As verrugas foram removidas por curetagem. Não foi usada crioterapia por não estar disponível no serviço. A electrocoagulação foi usada em alguns casos de papilomas e outras pequenas lesões sem necessidade de exame histológico. Todas as lesões suspeitas foram conservadas em formol e enviadas para exame histológico. Foram considerados suspeitos os nevos de surgimento recente ou com alteração recente de aspecto, nomeadamente assimetria, bordos irregulares, coloração variada no mesmo nevo ou coloração negra, diâmetro maior que 6 $\mathrm{mm}$, prurido, ulceração e sangramento. Todos os doentes com cancro confirmado foram referenciados ao Instituto Português de Oncologia do Porto (IPO-Porto), exceptuando os casos de carcinoma baso-celular com margens livres iguais ou superiores a $2 \mathrm{~mm}$.

\section{Registos}

Todos os procedimentos foram registados em processo informático SAM e codificados pelo ICPC-2 (Quadro I). Também foi feito o registo em ficheiro EXCEL com identificação, idade, número do processo, médico de família, diagnóstico clínico, diagnóstico histológico, complicações e destino do utente, para análise descritiva.

\section{Critérios de admissão e de exclusão}

Foram avaliados todos os utentes com lesões suspeitas ou incómodas sempre que solicitado pelo próprio ou pelo respectivo médico de família. Foram admitidos todos os utentes com lesões dermo-epidérmicas e subcutâneas com menos de três centímetros, móveis em relação aos planos profundos e cuja localização permitisse o encerramento primário por sutura simples. Foi tido em consideração o consentimento e a preferência do utente de realizar a intervenção no centro de saúde. As crianças com menos de 10 anos só foram admitidas para tratamento de lesões superficiais desde que tolerassem a anestesia local e mediante consentimento dos pais. Foram excluídos os utentes com lesões maiores que três centímetros, lesões fixas aos planos profundos, lesões localizadas nas pálpebras ou outros locais próximos de grandes vasos ou nervos. Foram também excluídos os utentes hipocoagulados, insuficientes cardíacos, renais e hepáticos graves, com antecedentes de cicatrização hipertrófica ou quelóides, atrofia cutânea ou cicatrização deficiente (esclerodermia, hipoproteinémia, porfirias), portadores de pacemaker (contraindicação para o uso de electrocoagulador), doentes agitados e pouco colaborantes e doentes com história de alergia ou intolerância a anestésicos locais.

\section{RESULTADOS}

Foram intervencionados 282 utentes dos quais 164 (58\%) do sexo feminino, incluídos em todas as faixas etárias, com predomínio entre os 15 e os 59 anos (Figura 1). Do total de utentes, 40 pertenciam à lista de utentes da autora, 193 de outras listas, e 49 foram utentes sem médico de família (Figura 2).

Foram realizados 341 procedimentos (em alguns utentes foi realizada a exérese de mais do que uma lesão), dos quais $170(49,8 \%)$ com recurso a exame histológico para confirmação diagnóstica. No caso dos papilomas cutâneos, foi registado como uma lesão por utente independentemente do número de lesões. As 


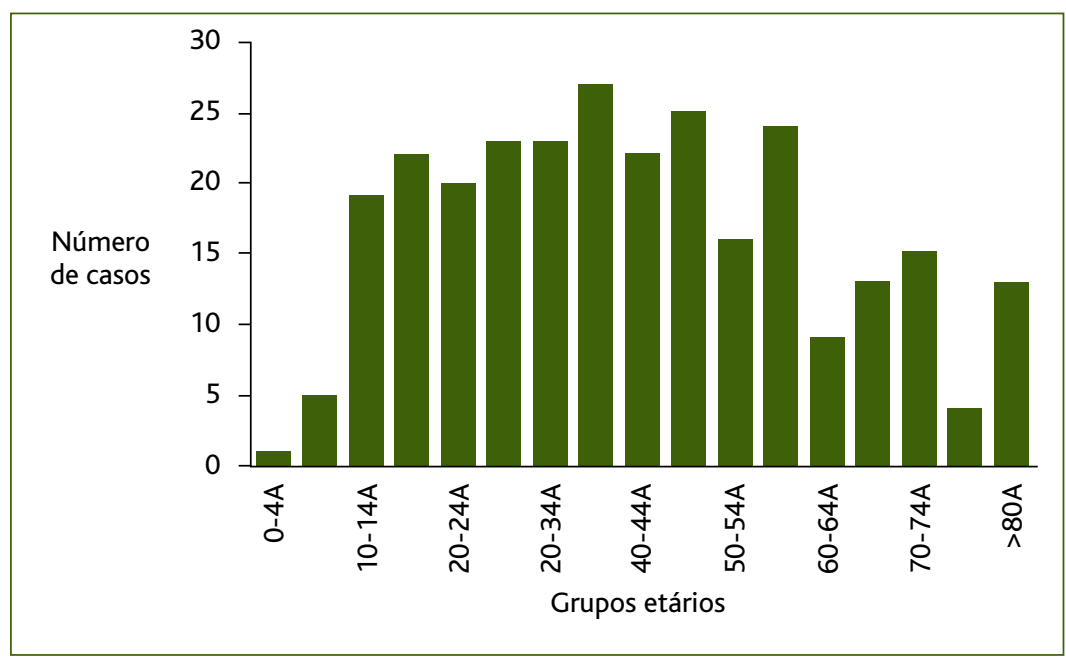

Figura 1. Distribuição dos utentes intervencionados no Centro de Saúde de Amarante por grupos etários.

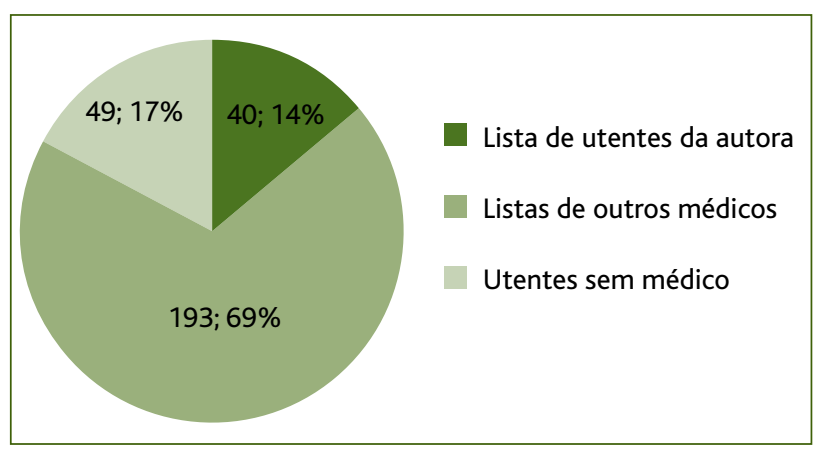

Figura 2. Distribuição dos utentes intervencionados no Centro de Saúde de Amarante por listas de utentes.

lesões mais comuns foram (Quadro II): quisto epidérmico (61 casos), nevo melanocítico dérmico (47 casos), nevo melanocítico composto (27 casos), queratose seborreica (28 casos) e papilomas cutâneos (27 casos). Foram também identificadas as seguintes lesões précancerosas: queratose solar actínica (8 casos), corno cutâneo ( 2 casos), lesão induzida por HPV (1 caso), nevo melanocítico atípico ( 7 casos) e nevo melanocítico congénito ( 2 casos). Além disso, encontraram-se as seguintes lesões cancerosas: carcinoma espinocelular in situ (1 caso), carcinoma basocelular (3 casos) e melanomas ( 3 casos).

Em todos os casos de lesões pré-cancerosas foi confirmada histologicamente a exérese com margens livres e todos os casos de lesões cancerosas foram refe- renciados aos cuidados secundários (IPO-Porto) para seguimento. Dos 3 casos de melanoma, um foi identificado numa mulher de 71 anos, que apresentava um nevo ulcerado e inflamado na perna. O segundo caso foi identificado num homem de 31 anos, que apresentava um pequeno nevo de cor negra na região sub-mentoniana de surgimento recente. E o terceiro caso foi identificado numa rapariga de 16 anos, que apresentava um nevo de cor negra e crescimento recente no terceiro dedo do pé direito. Em todos eles foi feita a revisão da cicatriz e confirmada exérese completa com ausência de metástases pelo IPO-Porto.

Todos os utentes foram acompanhados até à remoção da sutura ou à finalização de curativos pela enfermeira assistente e todas as complicações foram registadas e tratadas. Verificaram-se quatro complicações pós-operatórias $(0,01 \%)$, nomeadamente, dois casos de formação de quelóide, um caso de deiscência de sutura (região interescapular) e um caso de infecção pós-operatória.

\section{DISCUSSÃO}

Estes resultados não reflectem a taxa de incidência esperada de cancro cutâneo na população do Concelho de Amarante nem era este o propósito deste trabalho. De qualquer modo, esperava-se encontrar mais casos de carcinoma espinocelular e carcinoma basocelular do que de melanomas. Este facto pode dever-se a uma maior preocupação da população mais jovem relativamente aos «sinais» e suas alterações ou mesmo efeito estético; enquanto a população mais idosa, em que os epiteliomas são mais frequentes, se encontrem menos sensibilizados, atribuindo a desculpa da velhice às lesões que vão surgindo. Provavelmente a incidência será bem maior pelo facto de uma grande parte da população desta região viver do trabalho agrícola e por isso estar muito tempo exposta ao sol.

A taxa de infecções pós-operatórias foi insignificante, embora as condições de assepsia não fossem as ideais. Isto deve-se provavelmente ao facto de o ambiente local ser menos conspurcado por bactérias mul- 


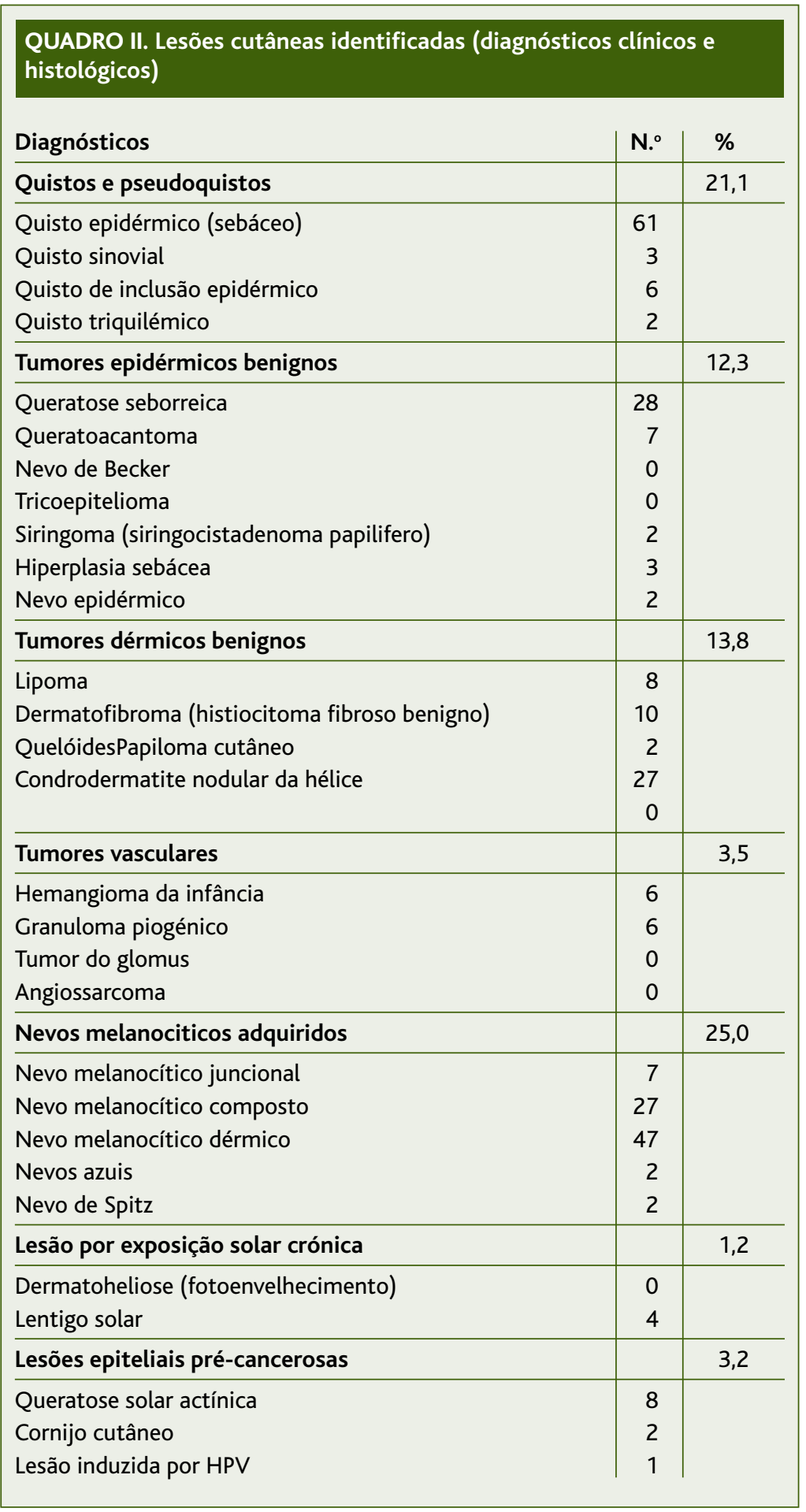

aproximação deste serviço à comunidade, para além da redução do tempo de espera e da redução da distância ao hospital de referência.

Esta experiência mostra que é possível estender a detecção precoce e o tratamento de neoplasias cutâneas aos Cuidados de Saúde Primários. Esta prática está muito generalizada na Austrália, Espanha e no Reino Unido. A Austrália é o país com a maior prevalência mundial de neoplasias cutâneas e onde a maior parte dos doentes são diagnosticados e tratados por clínicos gerais. ${ }^{9,10}$ Num estudo publicado em 2008, Heal et al demostram que os clínicos gerais da região de Queensland têm competência comparável à dos dermatologistas do diagnóstico clínico de lesões com potencial maligno. Isto se deve à sua larga experiência associada a uma certificação pelo Australasian College of Skin Cancer Medicine. ${ }^{11,12} \mathrm{~A}$ British MedicalAssociation considera que os procedimentos de pequena cirurgia realizados por clínicos gerais têm um alto custo-benefício e resultam em altos níveis de satisfação dos utentes. ${ }^{13} \mathrm{O}$ documento "Our health, our care, our say: a new direction for community services», publicado em 2006 no Reino Unido, preconiza o futuro dos cuidados de saúde fora dos hospitais. ${ }^{14}$ Reforça a importância dos serviços prestados por profissionais de saúde próximos da comunidade. Neste contexto, foi elaborado o documento «Guidance and Competencies for provision of services using GPs with Special Interests (GPwSIs) - Dermatology and Skin Surgery", em conjunto com representantes da British Association of Dermatologists, Primary Care Dermatology Society, Skin Care Campaign e Royal College of General Practitioners, que descreve diferentes modelos de serviços de der-

tirresistentes como acontece em meio hospitalar. Isto pode ser considerado como mais uma vantagem da matologia, formação, treino e creditação de clínicos gerais com interesse em cirurgia dermatológica. ${ }^{15,16}$ 


\begin{tabular}{|c|c|c|}
\hline \multicolumn{3}{|l|}{ QUADRO II. (continuação) } \\
\hline Diagnósticos & N..$^{\circ}$ & $\%$ \\
\hline Lesões epiteliais cancerosas & & 1,2 \\
\hline Carcinoma espinocelular in situ (Doença de Bowen) & 1 & \\
\hline Carcinoma espinocelular invasivo & 0 & \\
\hline Carcinoma basocelular & 3 & \\
\hline Lesões percursoras do melanoma & & 2,6 \\
\hline Nevo melanocítico atípico (Nevo displasico, Nevo de Clark) & 7 & \\
\hline Nevo melanocítico congénito & 2 & \\
\hline Lesões melanocíticas cancerosas & & 0,9 \\
\hline Melanoma & 3 & \\
\hline Outras & & 15,2 \\
\hline Unha encravada & 12 & \\
\hline Verrugas & 24 & \\
\hline Reação inflamatória de corpo estranho & 6 & \\
\hline Abcesso & 3 & \\
\hline Perioníquia & 3 & \\
\hline Foliculite crónica & 1 & \\
\hline Nevo pigmentado de Reed & 1 & \\
\hline Hematoma infectado & 1 & \\
\hline Neoplasia mesenquimatosa vascular incerta & 1 & \\
\hline Total & 341 & $100 \%$ \\
\hline
\end{tabular}

Classificação adaptada de «Fitzpatrick's Color Atlas \& Synopsis of Clinical Dermatology», Fifth Edition, 2005.

Em Portugal, a prática de pequena cirurgia em Cuidados de Saúde Primários tem-se tornado mais evidente com o surgimento das Unidades de Saúde Familiar que a incluem como carteira adicional de serviços. A Reforma dos Cuidados de Saúde prevê a diferenciação profissional resultante da emergência de novas competências que permitam uma estratégia de diversificação que vá ao encontro de novas necessidades e expectativas dos cidadãos. ${ }^{17}$ Assim, impõe-se, nos Cuidados de Saúde Primários, o aprofundamento de uma cultura de análise prospectiva de necessidades, tendo em vista a concepção e a promoção de «novos» produtos e serviços. É necessário promover o desenvolvimento de competências tendo em vista o rejuvenescimento da carteira básica e adicional de serviços com novos serviços e cuidados de saúde que resultem da integração de abordagens multidisciplinares e da aposta em soluções com vocação preventiva e curativa. Tam- bém é importante desenvolver protocolos com vista à estandardização e homogeneização destes serviços, tendo em conta critérios de qualidade, segurança $\mathrm{e}$ eficiência.

A nossa experiência, que iniciou de forma ocasional, sem qualquer tipo de publicidade, tornou-se rapidamente muito popular, com grande adesão por parte dos utentes. O facto de reduzir tempos de espera e de encurtar distâncias relativamente aos hospitais de referência desta região foi um factor determinante na preferência dos utentes. Existem fortes evidências que a prática continuada de exérese de lesões melhora a capacidade do médico de fazer rastreios clínicos, evitando assim tratamentos e biópsias desnecessárias. Melhora também a relação dos utentes com o seu médico de família e a proximidade com o seu centro de saúde. Por outro lado, descongestiona os serviços hospitalares ao limitar as referenciações a situações com diagnósticos comprovados e/ou de maior gravidade.

\section{AGRADECIMENTOS}

Os autores desejam agradecer à Dra. Maria Rosa Coelho pelo apoio e incentivos organizacionais na execução dos procedimentos descritos neste trabalho e pela leitura crítica do texto.

\section{REFERÊNCIAS BIBLIOGRÁFICAS}

1. Instituto Português de Oncologia do Porto, EPE. Registo Oncológico 2008. Porto: Centro Regional de Oncologia do Porto; 2008.

2. Liga Portuguesa Contra o Cancro. Cancro da pele não Melanoma. Disponível em: http://www.ligacontracancro.pt/gca/index.php?id=19 [acedido em 25/06/2010].

3. Silva HP, Ferrão C. Cancro da pele: podemos preveni-lo? Rev Port Clin Geral 2003 Set-Out; 19 (5): 485-90.

4. U.S.Preventive Services Task Force. Screening for Skin Cancer. Recommendation Statement. February 2009. Disponível em: http://www.uspreventiveservicestaskforce.org/uspstf09/skincancer/skincanrs.htm [acedido em 26-06-2010].

5. Jerant AF, Johnson JT, Sheridan CD, Caffrey Tj. Early detection and treatment of skin cancer. Am Fam Physician 2000 Jul 15; 62 (2): 357-68, 375-6, 381-2.

6. Lemos P. Enquadramento Nacional e Internacional da Cirurgia de Ambulatório. Hospitais SA. Unidade de Missão. Disponível em: http://www.hospitaisepe.min-saude.pt/NR/rdonlyres/70A32A38- 
1752-4874-B892-OBDD23BBDBB0/4075/EnquadramentonacionaleinternacionaldaCA.pdf [acedido em 25-06-2010].

7. Vaquero Martinez JJ, García Aparicio JM, Díaz Gómez J, Blasco paredes D. Eficiencia de la cirugía menor en atención primaria según el nivel de facturación. Aten Primaria 2002 Jun 30; 30 (2): 86-91.

8. Dixon AJ, Hall RS. Managing skin cancer: 23 golden rules. Aust Fam Physician 2005 Aug; 34 (8): 669-71.

9. Heal C, Buettner P, Raasch B, Browning S. Minor skin excisions in general practice in North Queensland. Aust Fam Physician 2006 Oct; 35 (10): 825-8.

10. Byrnes $P$, Ackermann E, Williams ID, Mitchell GK, Askew D. Management of skin cancer in Australia: a comparison of general practice and skin cancer clinics. Aust Fam Physician 2007 Dec; 36 (12): 1073-5.

11. Heal CF, Raasch BA, Buettner PG, Weedon D. Accuracy of clinical diagnosis of skin lesions. Br J Dermatol 2008 Sep; 159 (3): 661-8.

12. Dixon A, Rosengren $\mathrm{H}$, Connellly $\mathrm{T}$, Dixon J. Education in skin cancer management: assessing knowledge and safety. Aust Fam Physician 2009 Jul; 38 (7): 557-60.

13. Minor Surgery - Specification for a directed enhanced service. British Medical Association. Standing up for Doctors. 31 July 2007. Disponível em: http://www.bma.org.uk/employmentandcontracts/independent_contractors/enhanced_services/DESsurgery.jsp [acedido em 27/06/2010].

14. Our Health, our care, our say: a new direction for community services. Department of Health, January 2006. Disponível em: http://www.northwest.nhs.uk/document_uploads/Maternity_Matters/our_health_our_c are_our_say.pdf [acedido em 27/06/2010].

15. General practitioners with special interests, January 2006. Royal College of General Practitioners. Disponível em URL: http://www.rcgp. org.uk/pdf/ISSinfo_11_Jan06.pdf [acedido em 27/06/2010].

16. Guidance and Competencies for The provision of services using GP's with special interests (GPwSIs). Dermatology and Skin Surgery. Department of health.2007. Disponível em: http://www.pcc.nhs.uk/uploads/pwsis/gpwsis-dermatology.pdf [acedido em 27/06/2010].

17. Reforma dos cuidados de Saúde Primários. Plano estratégico 20072009. Missão para os cuidados de Saúde primários. Disponível em: http://www.portugal.gov.pt/pt/documentos/Governo/MS/Estrategia_cuidados_Saude_Primarios.pdf [acedido em 27/06/2010].

\section{CONFLITOS DE INTERESSE}

Os autores declaram não possuir qualquer conflito de interesses. O trabalho relatado não foi objecto de qualquer tipo de financiamento externo.

\author{
ENDEREÇO PARA CORRESPONDÊNCIA \\ Ana Cristina Neves \\ Praceta António Coelho Figueiredo, 58, 5. DF \\ 4400-361 Vila Nova de Gaia \\ anacristinaneves.7@gmail.com
}

Recebido em 17/11/2010

Aceite para publicação em 08/07/2011

\section{ABSTRACT}

\section{EARLY DETECTION OF SKIN CANCER IN PRIMARY CARE - AN EXPERIENCE}

Background: Skin cancer has an elevated prevalence in Portugal. It is estimated that the incidence of melanoma is $8 / 100,000$ persons/year and the incidence of epitheliomas is 100/100,000 persons/year. The incidence of melanoma has been increasing by 6 to $7 \%$ per year. Twenty percent of patients with melanoma will die as a result of their disease and half of these patients will die before the age of 40 . The progressive increase in incidence is due not only to an increase in new cases, but also due an increase in consultations.

Methodology: This paper reports on a 22-month-long experience (July 2008 to May 2010) of the excision of small skin lesions through minimally invasive surgical procedures. 282 patients of all ages were treated. Of these $58 \%$ (164) were female. A total of 341 procedures were performed. Histological examination for diagnostic purposes was performed in $49.8 \%$ (170).

Results: The most common lesions observed were epidermal cysts, intradermal melanocytic nevi and compound melanocytic nevi. The following pre-cancerous lesions were also observed: actinic keratosis, cornu cutaneum, HPV lesions, atypical melanocytic nevi, and congenital melanocytic nevus. The following cancerous lesions were observed and excised: squamous cell carcinoma in situ, basal cell carcinoma and melanoma. In all cases of precancerous lesions, complete excision with clear margins was confirmed by histology. All cases of cancerous lesions were referred to secondary care for follow-up and continued treatment. Discussion: It is possible to perform early detection and treatment of skin cancer in primary care. The accessibility of this service is appreciated by patients, allowing for shorter distances travelled to obtain care and reduced waiting times. In future studies, cost-effectiveness and impact on the health of the population should be addressed.

Keywords: Minor Surgery; Skin Cancer. 